Valentina D. A. Corino*, Luca lozzia, Giorgio Scarpini, Luca T. Mainardi and Federico Lombardi

\title{
A simple model to detect atrial fibrillation via visual imaging
}

https://doi.org/10.1515/bmt-2019-0153

Received June 17, 2019; accepted February 21, 2020
Abstract: Automatic detection of atrial fibrillation (AF) is a challenging issue. In this study we proposed and validated a model to identify AF by using facial video recordings. We analyzed photoplethysmographic imaging (PPGi) signals, extracted from video of a subject's face. Sixty-eight patients were included: 30 in sinus rhythm (SR), 25 in $\mathrm{AF}$ and 13 presenting with atrial flutter or frequent ectopic beats (ARR). Twenty-six indexes were computed. The dataset was divided in three subsets: the training, validation, and test set, containing, respectively, 58,29 , and $13 \%$ of the data. Mean of inter-systolic interval series (M), Local Maxima Similarity (LMS), and pulse harmonic strength (PHS) indexes were significantly different among all groups. Variability and irregularity parameters had the lowest values in SR, the highest in $\mathrm{AF}$, with intermediate values in ARR. The PHS was higher in SR than in ARR, and higher in ARR than in AF. The LMS index was the highest in SR, intermediate in ARR and the lowest in AF. Similarity indexes were higher in $\mathrm{SR}$ than in AF and ARR. A model with three features, namely M, Similarity1 and LMS was chosen. With this model, the accuracy for the validation set was $0.947 \pm 0.007$ for SR, $0.954 \pm 0.004$ for AF and $0.919 \pm 0.006$ for ARR; for the test set (never-seen data), accuracy was $0.876 \pm 0.021$ for SR, $0.870 \pm 0.030$ for $\mathrm{AF}$ and $0.863 \pm 0.029$ for ARR. A contactless video-based monitoring can be used to detect $\mathrm{AF}$, differentiating it from $\mathrm{SR}$ and from frequent ectopies.

Keywords: atrial fibrillation; camera; monitoring; photoplethysmographic signal; screening.

*Corresponding author: Valentina D. A. Corino, Department of Electronics, Information and Bioengineering, Politecnico di Milano, via Ponzio 34, 20133 Milan, Italy, E-mail: valentina.corino@polimi.it Luca lozzia and Luca T. Mainardi: Department of Electronics, Information and Bioengineering, Politecnico di Milano, Milan, Italy Giorgio Scarpini and Federico Lombardi: Fondazione IRCCS Ca Granda Ospedale Maggiore Policlinico, U.O.C. di Malattie Cardiovascolari, Università degli Studi di Milano, Dipartimento di Scienze Cliniche e di Comunità, Milan, Italy

\section{Introduction}

Atrial Fibrillation (AF) is often asymptomatic [1] thus making arrhythmia recognition difficult or impossible in many patients. The incapacity to diagnose AF in time has important clinical implications, since even brief episodes of asymptomatic $\mathrm{AF}$ are associated with increased risk of stroke, heart failure, hospitalization, and death [2]. The thromboembolic risk increases significantly when AF duration exceeds $48 \mathrm{~h}$ [3]. The impact is also economical since the costs related to health care resources utilization increase.

The recent guidelines of the European Society of Cardiology stress the role of opportunistic screening to improve AF detection [4] in all subjects older than 60 year of age. This indication is apparently supported by recent technological advances that may allow a better and more frequent monitoring of risk patients as well as a screening of general population, using widespread devices such as arm-cuff manometers and smartphone [5, 6]. Several wristband devices are now available over the counter. They are based on the recording and analysis of a photoplethysmographic (PPG) signal obtained by direct contact. The PPG signal, showing high variability and irregularity during AF, has been shown to well differentiate AF from sinus rhythm (SR). Other arrhythmias (ARR), such as atrial flutter or tachyarrhythmias, can also be identified [7].

It has been shown that by recording an individual's face and extracting the subtle beat-to-beat variations of skin color, it is possible to obtain a signal (the PPG imaging, photoplethysmographic imaging (PPGi)) that reflects cardiac pulsatile signal $[8,9]$. This technology could be of particular interest in those clinical conditions where traditional electrocardiogram (ECG) recordings are difficult to obtain: neonates, people with skin damage, and very elderly patients. A proof-of-concept study [10] has demonstrated the feasibility of AF detection by the use of a contactless technology, recording an individual's face and extracting the subtle beat-to-beat variations of skin color, reflecting the cardiac pulsatile signal. This study included only 11 patients and introduced an index quantifying the pulse variability, called the pulse harmonic strength (PHS) that resulted associated with a $20 \%$ detection error rate. 
We have recently reported the feasibility of this methodology of detecting AF [11]. Aim of this study is to propose and validate a model to identify AF by using the PPGi, i. e., by analyzing face color changes due to blood volume pulse (BVP) variations, differentiating it not only from SR, but also from other rhythm disturbances.

\section{Materials and methods}

\section{Study design}

Patients admitted to the Cardiovascular Diseases Unit of the Ospedale Maggiore Policlinico in Milan, Italy were enrolled. The study was approved by the internal Ethical Committee of the Hospital. During the procedure, an RGB (red, green and blue) camera was positioned on a tripod, placed in front of the subject at a distance of $1.5 \mathrm{~m}$ and connected to a PC workstation. The camera recorded the face of the patient for the whole session of the experiment ( $3 \mathrm{~min}$ ). During the acquisition, a 12-lead ECG was recorded so that an expert cardiologist could determine the type of rhythm. Possible rhythm classes were SR, AF, and ARR.

As video recording device, an industrial camera (GigE Sony XCGC30C camera) was used with spatial resolution of $659^{\star} 494$ pixels. Videos were acquired with a frame-rate of 120 FPS and 8 bits resolutions. Data were saved in RGB, uncompressed, audio-video interlaced (AVI) raw format to be processed offline. The camera was equipped with $15 \mathrm{~mm}$ fixed focal length lenses.

Each patient was asked to sit on a chair and to move as little as possible. The ambient setting included sunlight coming from a window, placed in front of subject's face to avoid possible shadows on his face and an artificial light due to the room neon lamps.

\section{Patient characteristics}

Seventy-six patients were enrolled into the study between September 2016 and May 2017. According to cardiac rhythm classification, 25 subjects were in AF (14 males and 11 females, mean age of $73 \pm 13$ years), 33 subjects in SR ( 23 males and 10 females, mean age of $66 \pm 19$ years), and 18 subjects (ARR) presented either atrial flutter or frequent atrial or ventricular ectopies (eight males and 10 females, mean age $74 \pm 7$ years). Three subjects in SR were excluded from the study, since they were unable to remain in silence or to avoid moving their face during the whole duration of the acquisition. Five subjects with a pacemaker to control ventricular response to atrial flutter were excluded from the ARR group, as ventricular rhythm was not physiological but paced by the device. The clinical characteristics of the analyzed patients are shown in Table 1.

\section{Extracting plethysmographic imaging (PPGi) signal from facial video}

Videos, saved in RGB, AVI raw format, were processed offline to extract the beat-to-beat pulsations, according to a method previously validated [9], and briefly described in the following. First, three different regions of interest (ROIs) were recognized and tracked over time: forehead, nose and cheek. ROI detection was performed by the Viola-Jones face detection algorithm [12] while ROI tracking for horizontal and vertical movements was obtained by the Lucas-KanadeTomasi motion flow tracking algorithm [13]. Secondly, a spatial average of pixel intensity of each channel (red, green and blue) was computed for each ROI and for each frame to retrieve reflected light modulation directly correlated to the BVP changes of facial peripheral arteries. Finally, the signals were detrended [14] and band-pass filtered (cut-off frequencies fc1 $=0.1 \mathrm{~Hz}$ and fc $2=5 \mathrm{~Hz}$ ) to remove noise and to enhance pulsatile component.

Thus, three PPGi signals were obtained, one from each of the analyzed ROI. Among them, the selection of the target PPGi signal was achieved by calculating the power spectral density (PSD) on the entire signal and by measuring the signal-to-noise ratio $(S N R)$ using the following formula:

$$
S N R=10 * \log _{10}\left(\frac{\int_{f_{1}}^{f_{2}} P_{P P G i_{n}(f)} d f}{\int_{0.1}^{f_{1}} P_{P P G i_{n}(f)}} d f+\int_{f_{2}}^{4} P_{P P G i_{n}(f)} d f\right)
$$

where $P_{P P G i n}(\mathrm{f})$ (with $n$ varying from one to three in this study) is the PSD of $n$th PPGi signal, $\mathrm{f}_{1}=f p-0.15, \mathrm{f}_{2}=f p+0.15$ and where $f p$ is the

Table 1: Demographic characteristics and cardiovascular history in the study population.

\begin{tabular}{llll}
\hline Variable & \multicolumn{1}{c}{ NSR } & AF & ARR \\
\hline $\mathrm{N}$ & 30 & 25 & 13 \\
Gender (male/female) & $20 / 10$ & $12 / 13$ & $6 / 7$ \\
Age (years) & $64 \pm 19(24-86)$ & $74 \pm 13(38-89)$ & $74 \pm 7(65-82)$ \\
AF duration (months) & - & $34 \pm 42(0.5-132)$ & - \\
Previous AF & - & 4 & - \\
Previous electrical cardioversion & - & 9 & 1 \\
Previous myocardial infarction & 7 & 0 & 1 \\
Ejection fraction & $57 \pm 8(35-68)$ & $57 \pm 8(23-65)$ & $55 \pm 11(34-65)$ \\
Diabetes & 6 & 2 & 4 \\
Hypertension & 15 & 14 & 8 \\
Rate-control drugs & 0 & 16 & 5 \\
Amiodarone & 0 & 1 & 0 \\
\hline
\end{tabular}


pulse frequency (measured in $\mathrm{Hz}$ ). The signal with the highest SNR was selected as PPGi signal to be further analyze. The BVP systolic peaks were detected by the algorithm of Scholkmann et al. [15], and from them the inter-systolic interval series were computed.

\section{Processing of PPGi signal}

Twenty-six indexes were computed belonging to the following three classes: (i) Spectral analysis, (ii) Variability and Irregularity analysis, (iii) Shape analysis. Parameters from the first two classes were computed on the inter-systolic interval series, whereas shape analysis was performed on the PPGi signal.

In the spectral analysis, the Pulse Harmonic strength (PHS) was used [10] to measure the strength of fundamental frequency compared to the total energy of the total spectrum. PHS is defined as the energy of the fundamental frequency and its main harmonics within 0.05 and $3 \mathrm{~Hz}$ band width divided by the energy of the remaining spectral components.

Variability and irregularity quantify different properties: variability is related to the dispersion of data, whereas irregularity is related to the degree of unpredictability of the data fluctuations. Variability analysis of the inter-systolic intervals series includes the mean (M), the standard deviation (SD), the root of the mean squared differences of successive intervals, (rMSSD) and the percentage of interval differences of successive intervals greater than $x \mathrm{~ms}$ (pNNx, with $x=10,20, \ldots, p N N 100$ )[7]. An additional feature was extracted by Lorentz plot (Lor), assessing the spatial distribution of inter-systolic intervals in the scatter plot. Shortly, in the Lor, each RR interval is plotted as a function of the previous one [16]. Thus, the dispersion of point's perpendicular to the line-of-identity reflects the level of shortterm variability, while the dispersion along the line-of-identity indicates the level of long-term variability. Lor represents the ratio between the dispersion of point's perpendicular and along the line-ofidentity.

Irregularity of the inter-systolic intervals series was assessed by approximate (ApEn) [7] and sample entropy (SampEn) [7], that quantify the unpredictability of fluctuations. Approximate entropy (ApEn) is a regularity statistic quantifying the unpredictability of fluctuations in a time series. Intuitively, the presence of repetitive patterns of fluctuation makes it more predictable than a time series in which such patterns are absent. ApEn reflects the likelihood that similar patterns will not be followed by additional similar observations. A time series containing many repetitive patterns, i. e., a regular and predictable series, has a relatively small ApEn, while a less predictable, i. e., more complex process, has a higher ApEn. The computation of ApEn for any series of length $(\mathrm{N})$ starts with the choice of two parameters: the length of patterns to be compared, $\mathrm{m}$, and the tolerance of mismatch, $\mathrm{r}$, between the corresponding elements. $\operatorname{ApEn}(\mathrm{m}, \mathrm{r}, \mathrm{N})$ quantifies the number of similar patterns which will remain similar when a new sample is added (i. e., when the length of the pattern increase from $m$ to $m+1)$. SampEn is an improved version of ApEn, which does not consider self-matches of patterns, an inclusion that makes ApEn a biased estimator. Sample entropy has the advantage to converge more rapidly than ApEn and thus it can be safely computed on shorter series. To compute ApEn and SampEn, we used the classical parameters $\mathrm{r}=0.2 \times$ standard deviation and $\mathrm{m}=1$ and 2 .

A measure of similarity between waves was obtained by calculating the relative number of similar pairs of waves in the recording
[17]. Briefly, each wave is represented as a point of the p-dimensional real space; the normalized waves are points belonging to the $\mathrm{p}$ dimensional unitary sphere. Hence, the morphological dissimilarity between two waves is evaluated by using the standard metric of the sphere to compute their distance

$$
D_{i, j}=\arccos \left(\boldsymbol{w}_{i}^{N} \cdot \boldsymbol{w}_{j}^{N}\right)
$$

where $\boldsymbol{w}_{i}^{N}$ and $\boldsymbol{w}_{j}^{N}$ represent the $i$ th and $j$ th normalized waves, i. e., $\boldsymbol{w}_{i}^{N}=\boldsymbol{w}_{i} /\left\|\boldsymbol{w}_{i}\right\|$ and (.) the scalar product. A measure of similarity between waves is obtained by calculating the relative number of similar pairs of waves in the recording. The similarity depends on the threshold $\varepsilon$ used in evaluating the similarity, that is, two waves are considered to be similar when their distance is lower than epsilon. In this study, five different thresholds are tested: epsilon $=[\pi / 2, \pi / 4, \pi / 8$, $\pi / 16$, and $\pi / 32$ ], defining $\operatorname{Sim} 1, \operatorname{Sim} 2, \operatorname{Sim} 3, \operatorname{Sim} 4$, and $\operatorname{Sim} 5$. A final index called Local Maxima Similarity (LMS) based on the similarity of local maxima distribution between consecutive temporal windows of the signal was introduced (Italian patent application "cardiac activity measurement system and method thereof" filed on May 04, 2018 with number 102018000005089).

\section{Statistical analysis}

Kruskal-Wallis one-way analysis of variance was performed to compare the computed parameters during SR, AF, and ARR. If the pvalue of the Kruskal-Wallis test was significant, an unpaired Wilcoxon test with Holm's correction was applied. A p $<0.05$ was considered statistically significant.

To create a model able to classify a patient rhythm according to his/her PPGi, feature selection and classification were performed. Data were divided into three groups: training and validation sets, to choose the best model, and a test set, to test the model on never-seen data. First, the test set was created, by randomly selecting data from nine patients (three for each rhythm). The remaining data (27 in SR, 22 in AF, and 10 in ARR) were used to select the best model. However, we observed that the dataset was imbalanced (i. e., the classes are not approximately equally represented), and this imbalance may produce classifiers with poor predictive accuracy for the minority class. Thus, we re-sampled the original dataset, by oversampling the minority classes using the synthetic minority over-sampling technique (SMOTE) [18], i. e., by creating synthetic examples.

As features selection procedure, a sequential forward floating search (SFFS) algorithm was used to identify the subset of optimal features. Briefly, the SFFS algorithm [19] starting from the empty set of features, the feature $x_{\mathrm{i}}$ that maximizes the objective function $\mathrm{J}$ when combined with the $\mathrm{k}$ features that have already been selected (Yk) is added (forward step). The objective function values with different number of features $J(k)$ is memorized, where k indicates the number of features. After the forward step, a backward step is performed. The backward step consists in removing from $\mathrm{Yk}$ the feature that makes the objective function $\mathrm{J}^{\star}(\mathrm{k})$ larger than $\mathrm{J}(\mathrm{k})$, where $\mathrm{J}^{\star}(\mathrm{k})$ is the objective function after removing one feature. The backward step is repeated as long as $\mathrm{J}^{\star}(\mathrm{k})$ is larger than $\mathrm{J}(\mathrm{k})$, with $\mathrm{k}$ decreasing, with the constrain that the last added feature cannot be removed. In this study, the objective function was the average of the accuracy for AF and for ARR, to decrease the false negative rate. Leave-p-out cross-validation was performed with 50 bootstrap repetitions, i. e., all the above steps are repeated 50 times. Accuracy (the ratio between the true results and the total number of cases), precision (or positive predictive value, i. e., the 
ratio between the true positive cases and all the predicted positive cases), and recall (or true positive rate, i. e., the ratio between the true positive cases and all the positive cases) were computed to evaluate the model. Accuracy, precision and recall were averaged over the total repetitions. The above procedure was repeated 30 times to assess the stability of the chosen features, using the Tanimoto index [20]. Using the average accuracy and the Tanimoto index computed on the validation set, the best model is chosen and then tested on the test set data (never-seen-data), created for each of the 50 repetition, thus obtaining an average test accuracy. The classification workflow is shown in Figure 1.

All analyses and statistical tests were performed using MATLAB R2018a (The MathWorks, USA).

\section{Results}

\section{PPGi characterization}

Table 2 shows the median (interquartile range) of all the computed parameters, together with the $\mathrm{p}$-value of the Kruskal-Wallis test and, if the p-value is significant, which comparisons were found statistically significant (Holm correction). Almost all the indexes were able to significantly differentiate AF from SR. However, only three parameters were significantly different among all groups of patients, namely the mean of the inter-systolic interval series (M), the LMS and the PHS. It is worth noting that these three parameters are based on different computational algorithms based on the analysis of, respectively, variability of the inter-systolic interval series, morphology of the PPGi signal and frequency analysis of PPGi signal.

Variability and irregularity parameters had the lowest values in $\mathrm{SR}$, and the highest in $\mathrm{AF}$, with intermediate values in the ARR group. The PHS was significantly different in the three rhythms, being higher in SR than in ARR, and higher in ARR than in AF. The LMS index was the highest in SR, intermediate in ARR and the lowest in AF. Signal similarity indexes were higher in SR than in AF and ARR, being quite similar in the two arrhythmias groups.

\section{Rhythm classification}

The classifier was trained with different sets of features, with an increasing number of features selected by the SFFS algorithm and the analysis was run 30 times to assess feature stability.

To choose the best model, average accuracy on the validation set as well as the Tanimoto index were taken into account. As shown in Figure 2a, the average accuracy for the three rhythms increases with the number of features reaching almost a constant value after five features, whereas the Tanimoto index is equal to one for the model using one or two features, then decreases and increases again after 10 features. To balance between accuracy and stability of the selected features, a model with three features was selected. Figure $2 \mathrm{~b}$ shows the percentage of stability of the selected features: M and LMS were always selected, while Similarity1 and Similarity2 were selected 73 and $27 \%$ of the times. Therefore, the final selected model is a model with three features, namely M, Similarity1 and LMS.

Table 3 (upper part) shows the accuracy, precision and recall for rhythm detection using the final selected model on the validation and test (never-seen data) sets. It can be noted that for the validation set, the accuracy was higher than 0.92 for all the rhythms, whereas it was about 0.87 for the test set. As for AF detection, recall was 0.915 in the test set, at the expenses of a precision of 0.752 ; on the contrary for ARR, precision was $0.875 \pm 0.035$ in the test set, at the expenses of a recall of $0.686 \pm 0.077$. For screening and identification of patients with arrhythmias, high recall

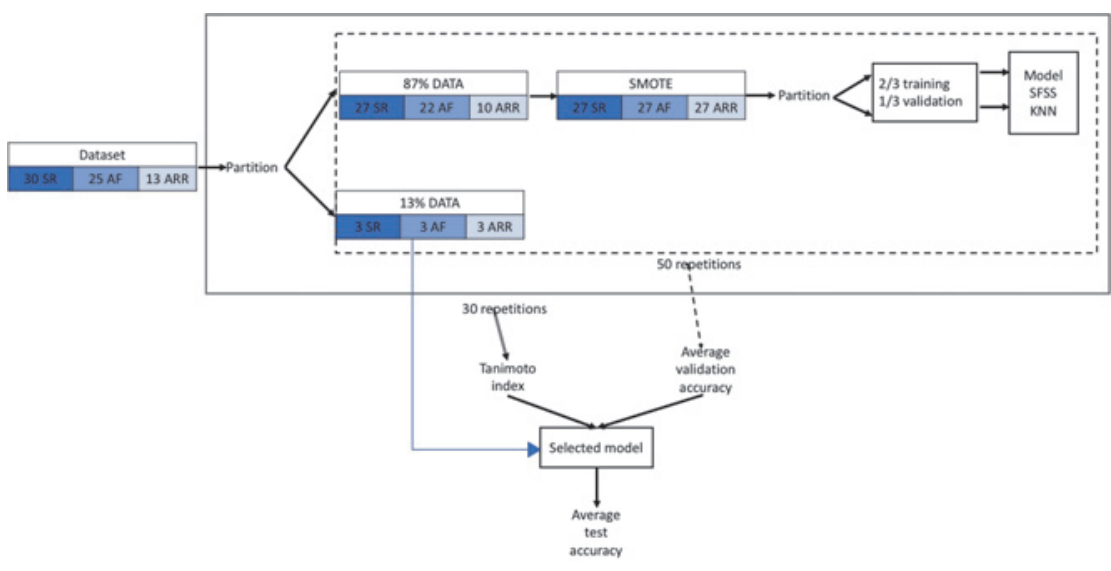

Figure 1: Schematic representation of the overall method. As first step, $13 \%$ of the data (three patients per group) are removed to be used as test group. The remaining $87 \%$ of the data enter the oversampling (obtained using synthetic minority oversampling technique (SMOTE)) and then the feature selection and classification algorithms, using $2 / 3$ of the data as training and $1 / 3$ as validation group. The classification algorithm gives as output the optimal model to be used on the test group. 
Table 2: All the computed parameters in the three groups, median (25th-75th percentiles).

\begin{tabular}{|c|c|c|c|c|c|}
\hline & SR & AF & ARR & p-Value KW & Pairwise significance \\
\hline$M(\mathrm{~ms})$ & 937 (815-1002) & 757 (692-831) & $680(586-713)$ & $p<0.001$ & Al \\
\hline $\mathrm{HR}(\mathrm{bpm})$ & $64(60-74)$ & $79(72-87)$ & $88(84-103)$ & $p<0.001$ & Al \\
\hline $\mathrm{SD}(\mathrm{ms})$ & $135(109-162)$ & $180(165-197)$ & $147(115-177)$ & $\mathrm{p}<0.001$ & SR vs. AF \\
\hline $\mathrm{SDD}(\mathrm{ms})$ & $206(177-246)$ & $241(224-262)$ & $199(142-231)$ & 0.02 & SR vs. $A F$ \\
\hline pNN10 (\%) & $92(90-94)$ & $94(93-96)$ & $94(89-95)$ & 0.02 & SR vs. AF \\
\hline pNN20 (\%) & $88(85-90)$ & $91(90-93)$ & $89(80-91)$ & 0.002 & SR vs. AF \\
\hline pNN30 (\%) & $84(79-87)$ & $88(86-91)$ & $85(71-87)$ & 0.002 & SR vs. AF \\
\hline pNN40 (\%) & $80(72-83)$ & $84(82-87)$ & $81(64-84)$ & 0.002 & SR vs. AF \\
\hline pNN50 (\%) & $76(66-80)$ & $81(77-85)$ & $78(56-80)$ & 0.002 & SR vs. $A F$ \\
\hline pNN60 (\%) & $72(62-77)$ & $79(74-82)$ & $73(49-77)$ & 0.001 & SR vs. $A F$ \\
\hline pNN70 (\%) & $67(58-74)$ & $75(71-80)$ & $69(44-73)$ & $\mathrm{p}<0.001$ & $S R$ vs. $A F$ \\
\hline pNN80 (\%) & $63(53-70)$ & $71(67-77)$ & $66(40-71)$ & $\mathrm{p}<0.001$ & SR vs. AF \\
\hline pNN90 (\%) & $59(49-66)$ & $69(64-73)$ & $63(36-68)$ & $p<0.001$ & SR vs. AF \\
\hline pNN100 (\%) & $51(41-59)$ & $64(59-68)$ & $57(31-62)$ & $p<0.001$ & SR vs. AF \\
\hline $\mathrm{rMSSD}(\mathrm{ms})$ & $205(176-245)$ & $240(223-261)$ & $199(142-231)$ & 0.02 & . \\
\hline ApEn_m1 (a.u.) & $1.6(1.5-1.7)$ & $1.8(1.8-1.9)$ & $1.7(1.5-1.8)$ & $\mathrm{p}<0.001$ & SR vs. $A F$ \\
\hline ApEn_m2 (a.u.) & $1.1(0.99-1.1)$ & $1.2(1.1-1.2)$ & $1.2(1.1-1.2)$ & $\mathrm{p}<0.001$ & $\begin{array}{l}\text { SR vs. AF } \\
\text { SR vs. ARR }\end{array}$ \\
\hline SampEn_m1 (a.u.) & $1.6(1.4-1.7)$ & $1.9(1.8-1.9)$ & $1.6(1.4-1.8)$ & $p<0.001$ & $S R$ vs. $A F$ \\
\hline SampEn_m2 (a.u.) & $1.6(1.4-1.6)$ & $1.9(1.7-2)$ & $1.6(1.4-1.7)$ & $\mathrm{p}<0.001$ & $S R$ vs. $A F$ \\
\hline Similarity1 (a.u.) & $0.94(0.91-0.97)$ & $0.91(0.87-0.93)$ & $0.9(0.86-0.94)$ & 0.005 & SR vs. $A F$ \\
\hline Similarity2 (a.u.) & $0.61(0.52-0.75)$ & $0.49(0.43-0.55)$ & $0.49(0.41-0.6)$ & 0.006 & SR vs. AF \\
\hline Similarity3 (a.u.) & $0.18(0.13-0.24)$ & $0.13(0.1-0.19)$ & $0.15(0.11-0.22)$ & ns & . \\
\hline Similarity4 (a.u.) & $0.021(0.013-0.043)$ & $0.018(0.013-0.032)$ & $0.025(0.015-0.049)$ & ns & - \\
\hline Similarity5 (a.u.) & $0.0013(0.0006-0.0031)$ & $0.0014(0.00082-0.003)$ & $0.0026(0.001-0.0068)$ & ns & - \\
\hline LMS (a.u.) & $0.94(0.88-0.96)$ & $0.77(0.71-0.8)$ & $0.86(0.77-0.92)$ & $\mathrm{p}<0.001$ & All \\
\hline PHS (a.u.) & $13(11-17)$ & $1(-1-2)$ & $3(1-10)$ & $\mathrm{p}<0.001$ & All \\
\hline Lor (a.u.) & $0.5(0.47-0.52)$ & $0.61(0.58-0.64)$ & $0.6(0.57-0.65)$ & $\mathrm{p}<0.001$ & $\begin{array}{l}\text { SR vs. AF } \\
\text { SR vs. ARR }\end{array}$ \\
\hline
\end{tabular}

SR, normal sinus rhythm; AF, atrial fibrillation; ARR, other arrhythmias.

$K W$, Kruskal-Wallis one-way analysis of variance.
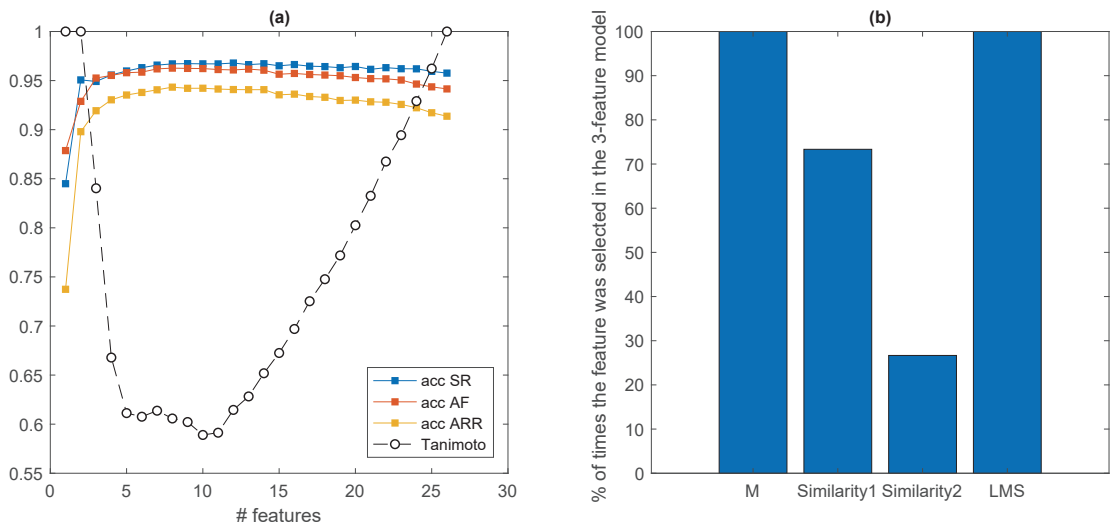

Figure 2: Choice of the best model. (a) The average accuracy for the three rhythms is shown together with the Tanimoto index as a function of the number of used features. (b) The percentage of stability of the selected features. might be preferable, as detection may lead to immediate treating of the arrhythmia.

Figure 3 shows the values for the three parameters selected by the SFSS and used in the model for all the patients, divided into the three rhythms. As all parameters overlap in the three groups, no single parameter could correctly classify the patient rhythm.

Figure 4 shows the 3D plot using the selected parameters as axes, as well as their projections with the three pairs of axes. It can be noted that, contrary to what 
Table 3: Accuracy, precision and recall for the three rhythms, using the model selected by the SFFS and the model with the three features able to significantly differentiate the three classes.

\section{SR}

$\mathrm{AF}$

2ARR

Model selected by the SFFS (M, Similarity1 and LMS)

Accuracy Validation Set

Accuracy Test Set

Precision Validation Set

Precision Test Set

Recall Validation Set

Recall Test Set

$0.947 \pm 0.007$
$0.876 \pm 0.021$
$0.922 \pm 0.013$
$0.816 \pm 0.036$
$0.912 \pm 0.013$
$0.813 \pm 0.033$

$0.954 \pm 0.004$

$0.919 \pm 0.006$

$0.870 \pm 0.030$

$0.863 \pm 0.029$

$0.910 \pm 0.012$

$0.899 \pm 0.012$

$0.752 \pm 0.047$

$0.875 \pm 0.035$

$0.958 \pm 0.010$

$0.853 \pm 0.018$

$0.915 \pm 0.026$

$0.686 \pm 0.077$

Model with the 3 features able to significantly differentiate the three classes (M, LMS and PHS)

Accuracy Validation Set

$0.959 \pm 0.007$

$0.876 \pm 0.012$

$0.923 \pm 0.008$

$0.903 \pm 0.009$

Accuracy Test Set

$0.939 \pm 0.015$

$0.815 \pm 0.014$

$0.792 \pm 0.015$

Precision Validation Set

$0.802 \pm 0.025$

$0.865 \pm 0.015$

$0.881 \pm 0.018$

Precision Test Set

$0.937 \pm 0.013$

Recall Validation Set

$0.835 \pm 0.033$

$0.672 \pm 0.018$

$0.786 \pm 0.032$

$0.822 \pm 0.020$

$0.526 \pm 0.044$
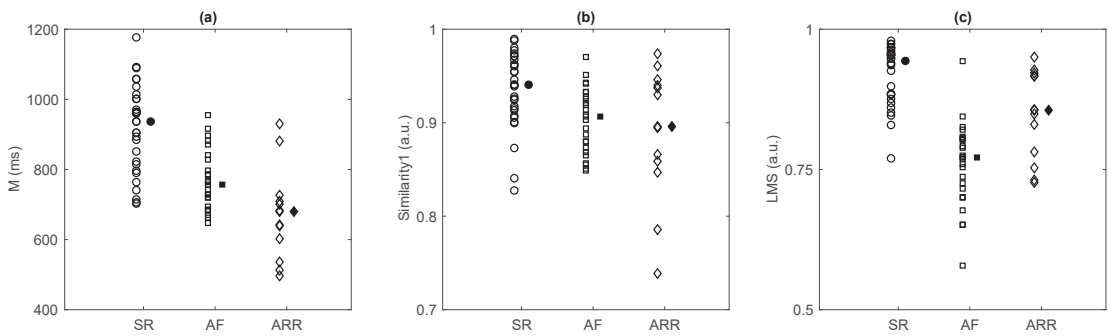

Figure 3: Parameters used in the model to predict cardiac rhythm. Values for the three parameters used in the model (a) mean of inter-systolic interval series $(M),(b)$ Similarity1, and (c) local maxima similarity (LMS) for all the patients, divided into the three rhythms (sinus rhythm (SR) circles, atrial fibrillation (AF) squares, other arrhythmias (ARR) diamonds). The filled markers show the median value in each group.

happens with a single parameter, the clouds of the three rhythms are well separated, with an accuracy of the model greater than $90 \%$.

We also investigated the performance of a model based on the three features that resulted able to significantly differentiate all the classes, i. e., M, LMS, and PHS (as stated in Section PPGi characterization). Table 3 (bottom part) shows the accuracy, precision and recall for the three rhythms using this model on the validation and test (neverseen data) sets used for the previous model. It is worth

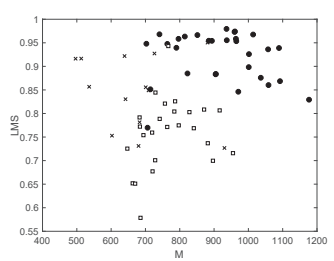

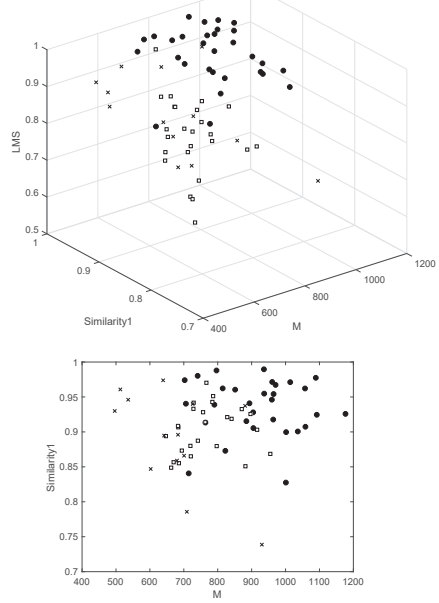

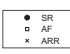

Figure 4: 3-D cloud of the parameters used in the model. 3D plot using M, Similarity1 and LMS as axes, divided into the three rhythms (SR circles, AF squares, ARR crosses). Their projections with the three pairs of axes are also shown. 
noting that the accuracy for SR was similar to that of the proposed model; whereas the accuracy for AF and ARR was significantly lower $(\mathrm{p}<0.05)$.

\section{Discussion}

The aim of this study was to create a model able to identify patients with $\mathrm{AF}$, by a simple short video of their face. Starting from a video of the face of a patient, using a validated technique [9], a PPGi signal is extracted and characterized by means of parameters assessing signal morphology and heart rate properties. The main finding is the possibility to accurately identify the rhythm of the subject and in particular to separate SR from AF, by using only three parameters computed on the PPGi signal.

Today, commonly available diagnostic tools are not sufficient to reveal all the patients with AF because of the silent nature of arrhythmia in a consistent proportion of patients [1]. In these subjects i. e., an asymptomatic form of AF incidentally can be diagnosed during routine examination or manifesting as an AF-related complication. The real incidence of silent $\mathrm{AF}$ in general population cannot be known, being this a major healthcare problem since silent $\mathrm{AF}$ has the same associated thromboembolic risk as symptomatic AF episodes [1, 21, 22]. Thus, the development of low cost and daily use monitoring devices is highly desirable. The proposed model is based on the PPGi technology, whose main advantage is the absence of contact between sensors and skin, thus not requiring patient active participation. In addition, once activated, video recording can also be programmed in absence of operator presence at preselected times.

This technology can be used for opportunistic detection of AF in several out of hospital conditions such as waiting rooms of family doctors. An additional area of potential use is prolonged monitoring of elderly subjects confined to bed as a result of neurological or orthopedic disorders or neonates or infants during hospital admission. Besides, it can be used in the ambulatory settings for a short-term evaluation of cardiac rhythm by simply asking the patients to look into the camera without necessity of a private environment as requested to record a traditional ECG.

To our knowledge, this is the first study aiming at detecting AF, differentiating it from SR and also from other arrhythmias using a contactless video of the subject's face. The technique we used to extract the PPGi has been validated before [9] and parameters computed on the extracted PPGi were successfully compared to those computed from the classical ECG [9]. The only previous study trying to detect $\mathrm{AF}$ from a video derived from a camera [10] included only 11 patients, whose face was recorded before and after cardioversion. They presented a proof-of-concept study designed to investigate monitoring of the pulsatile rate in humans using PPGi, showing that the PHS index was associated with a $20 \%$ detection error rate. In another study [23], 217 patients were included and facial photoplethysmography was recorded using an iPhone; pulse irregularity was evaluated and they reached an accuracy of 0.95 in detecting AF. In our study, we reached an average accuracy of 0.92 for AF (considering the validation and the test sets), differentiating AF from SR and from ARR too. This better result compared to [10] may be due to method used to classify the recordings, moving from a single parameter to a more complete model including three parameters describing both PPGi waveforms and VPGG rate. On the other hand, the worse result compared to [23] may be due to due to our aim, that is not only to differentiate $\mathrm{AF}$ from SR but also from ARR.

\section{Study limitations}

The limited number of patients enrolled in the study represents a major limitation. Observed results are however robust and the design of the study is based on training, validation, and testing phases. More controversial, for the present time and the future is the fact that the proposed methodology is based on video recording of subject face thus making all individuals easily identifiable. Recorded data however are analyzed off-line to derive PPGi whereas original videos can be deleted.

\section{Conclusion}

From the results of this study, a model based on a short video of a subject's face can be used to detect AF, differentiating it not only from SR but also from atrial flutter or frequent ectopic beats, thus being a promising tool to identify patients with AF. Further research is needed to determine the performance of this technology for opportunistic detection of AF in out of hospital conditions and in monitoring of subjects confined to bed.

\section{Research funding: None declared.}

Author contributions: All authors have accepted responsibility for the entire content of this manuscript and approved its submission.

Competing interests: Authors state no conflict of interest. Informed consent: Informed consent was obtained from all individuals included in this study. 
Ethical approval: The study conforms with the Declaration of Helsinki, and was approved by the Ethics Commit of Ospedale Maggiore Policlinico, Milan, Italy.

\section{References}

1. Dilaveris PE, Kennedy HL. Silent atrial fibrillation: epidemiology, diagnosis, and clinical impact. Clin Cardiol 2017;40:413-8.

2. Dries D, Exner D, Gersh B, Domanski M, Waclawiw M, Stevenson L. Atrial fibrillation is associated with an increased risk for mortality and heart failure progression in patients with asymptomatic and symptomatic left ventricular systolic dysfunction: a retrospective analysis of the SOLVD trials. J Am Coll Cardiol 1998;32:695-703.

3. Markides V. Atrial fibrillation: classification, pathophysiology, mechanisms and drug treatment. Heart 2003;89:939-43.

4. Benjamin EJ, Chen P, Bild DE, Alice M, Albert CM, Alonso A, et al. Prevention of atrial fibrillation: report from an NHLBI Workshop. Circulation 2009;119:606-18.

5. Krivoshei L, Weber S, Burkard T, Maseli A, Brasier N, Kühne M, et al. Smart detection of atrial fibrillation. Europace 2016:euw125. https://doi.org/10.1093/europace/euw125.

6. McManus DD, Chong JW, Soni A, Saczynski JS, Esa N, Napolitano C, et al. PULSE-SMART: Pulse-based arrhythmia discrimination using a novel smartphone application. J Ca vrdiovasc Electrophysiol 2016;27:51-7.

7. Corino VDA, Laureanti R, Ferranti L, Scarpini G, Lombardi F, Mainardi LT. Detection of atrial fibrillation episodes using a wristband device. Physiol Meas 2017;38:787-99.

8. Wu T, Blazek V, Schmitt H. Photoplethysmography imaging: a new noninvasive and noncontact method for mapping of the dermal perfusion changes. Proceedings volume 4163, optical techniques and instrumentation for the measurement of blood composition, structure, and dynamics. 2000. https://doi.org/10.1117/12. 407646REF.

9. Iozzia L, Cerina L, Mainardi L. Relationships between heart-rate variability and pulse-rate variability obtained from video-PPG signal using ZCA. Physiol Meas 2016;37:1934-44.

10. Couderc JP, Kyal S, Mestha LK, Xu B, Peterson DR, Xia X, et al. Detection of atrial fibrillation using contactless facial video monitoring. Hear Rhythm 2015;12:195-201.
11. Corino VDA, Iozzia L, Mariani A, D’Alessandro G, D’Ettorre C, Cerina $L$, et al. Identification of atrial fibrillation episodes using a camera as contactless sensor. In: 2017 Computing in cardiology (CinC). IEEE, Rennes, France; 2017. https://doi.org/10.22489/ CinC.2017.052-220REF.

12. Viola P, Jones M. Rapid object detection using a boosted cascade of simple features. In: Proceedings of the 2001 IEEE computer society conference on computer vision and pattern recognition. IEEE, Kauai, HI, USA; 2001:511-518.

13. Tomasi C, Kanade T. Detection and tracking of point features. United States: School of Computer Science Carnegie Mellon University; 1991.

14. Tarvainen MP, Ranta-aho PO, Karjalainen PA. An advanced detrending method with application to HRV analysis. IEEE Trans Biomed Eng 2002;49:172-5.

15. Scholkmann F, Boss J, Wolf M. An efficient algorithm for automatic peak detection in noisy periodic and quasi-periodic signals. Algorithms 2012;5:588-603.

16. Brennan M, Palaniswami M, Kamen P. Do existing measures of Poincaré plot geometry reflect nonlinear features of heart rate variability?. IEEE Trans Biomed Eng 2001;48:1342-7.

17. Faes L, Nollo G, Antolini R, Gaita F, Ravelli F. A method for quantifying atrial fibrillation organization based on wave-morphology similarity. IEEE Trans Biomed Eng 2002;49: 1504-13.

18. Chawla NV., Bowyer KW, Hall LO, Kegelmeyer WP. SMOTE: synthetic minority over-sampling technique. J Artif Intell Res 2002;16:321-57.

19. Pudil P, Novovičová J, Kittler J. Floating search methods in feature selection. Pattern Recognit Lett 1994;15:1119-25.

20. Kalousis A, Prados J, Hilario M. Stability of feature selection algorithms: a study on high-dimensional spaces. Knowl Inf Syst 2007;12:95-116.

21. Glotzer TV, Ziegler PD. Cryptogenic stroke: Is silent atrial fibrillation the culprit? Hear Rhythm 2015;12:234-41.

22. Guenancia C, Garnier F, Mouhat B, Béjot Y, Maillot N, Fichot M, et al. Dépistage et implications cliniques de la fibrillation atriale silencieuse. La Rev Médecine Interne 2018;39:574-9.

23. Yan BP, Lai WHS, Chan CKY, Chan SCH, Chan LH, Lam KM, et al. Contact-free screening of atrial fibrillation by a smartphone using facial pulsatile photoplethysmographic signals. J Am Heart Assoc 2018;7:e008585. 